\section{Kein Vorteil von Ticagrelor gegenüber Clopidogrel bei PAVK}

\author{
Im Gegensatz zu Patienten mit akutem Koronarsyndrom profitieren \\ Patienten mit symptomatischer peripherer arterieller Verschlusskrankheit \\ nicht von Ticagrelor gegenüber Clopidogrel.
}

$P^{\text {atinter }}$ tienten mit symptomatischer peripherer arterieller Verschlusskrankheit (PAVK) haben ein deutlich erhöhtes Risiko für kardiovaskuläre Ereignisse, vor allem bei mehreren betroffenen $\mathrm{Ge}$ fäßprovinzen. Seit der CAPRIE-Studie vor mittlerweile über 20 Jahren wird Clopidogrel gegenüber Acetylsalicylsäure als Plättchenhemmer der Wahl in der Sekundärprävention bei PAVK-Patienten mit stabiler kardiovaskulärer Erkrankung empfohlen. Studien konnten für Ticagrelor gegenüber Clopidogrel bei Patienten mit akutem Koronarsyndrom sowie nach zurückliegendem Myokardinfarkt eine Verringerung kardiovaskulärer Ereignisse bis hin zur Mortalitätsreduktion zeigen. Dies galt insbesondere auch für Patienten mit begleitender PAVK.
Ziel der nun publizierten, großen EUCLID-Studie war es daher, Ticagrelor gegen Clopidogrel an 13.885 Patienten mit PAVK zu vergleichen. Der kombinierte primäre Endpunkt beinhaltete den kardiovaskulären Tod, Myokardinfarkt und einen Schlaganfall. Die mittlere Nachbeobachtungsdauer betrug 30 Monate.

Im Ergebnis zeigte sich im primären Endpunkt kein signifikanter Unterschied zwischen den Behandlungsgruppen (Hazard Ratio 1,02; $\mathrm{p}=0,65$ ). Die Wahrscheinlichkeit kritischer Extremitätenischämien sowie die Wahrscheinlichkeit schwerer Blutungen unterschieden sich zwischen den Behandlungsgruppen ebenfalls nicht signifikant voneinander.
Hiatt WR et al. Ticagrelor versus Clopidogrel in Symptomatic Peripheral Artery Disease. N Engl J Med. 2017:376:32-40

\section{Kommentar}

Die EUCLID-Studie ist im Ausgang negativ. Ticagrelor als Monotherapie bietet keinen Vorteil gegenüber Clopidogrel bei PAVK. Es zeigte sich jedoch, dass sich das kardiovaskuläre Risikopotenzial und antithrombozytäre Behandlungergebnisse bei Patienten mit klinisch führender PAVK (und z.T. begleitender KHK) von jenen mit führender KHK (und z.T. begleitender PAVK) deutlich unterscheiden können. Aufgrund der weiterhin hohen Ereignisraten von Patienten mit signifikanter PAVK (4,5 MACE und 0,9\% kritische Beinischämie pro Jahr) sind weitere Studien mit klar definierten Patientenpopulationen notwendig.

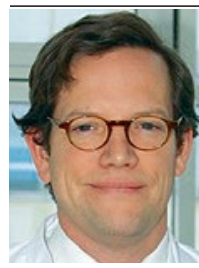

Prof. Dr. Peter W. Radke Klinik für Innere Medizin Kardiologie, Schön Klinik Neustadt

\section{Ausschreibung: Forschungspreis der DAG e.V. 2017}

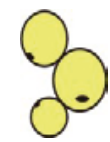

Auch in diesem Jahr schreibt die Deutsche Adipositas-Gesellschaft e.V. wieder einen Forschungspreis aus.

$\mathrm{D}$ ie Deutsche Adipositas-Gesellschaft (DAG) e. V. verleiht jedes Jahr auf ihrer Jahrestagung einen Forschungspreis für hervorragende wissenschaftliche Arbeiten im Bereich der AdipositasForschung. Sie will damit jüngere Wissenschaftler im deutschsprachigen Raum für deren herausragende wissenschaftliche Leistungen auszeichnen.

Der Forschungspreis der DAG e.V. hat ein hohes Ansehen in der Adipositas-Forschung in Deutschland. Die jährliche Vergabe des Preises ist ein zentrales Anliegen der Fachgesellschaft. Die Fachgesellschaft will damit auch junge Wissenschaftler fördern.

Als Beurteilungskriterien werden die bisherigen wissenschaftlichen Arbeiten auf dem Gebiet der Adipositas-Forschung sowie der wissenschaftliche Werdegang des Bewerbers herangezogen.

Der diesjährige Preis wird anlässlich der 33. Jahrestagung der DAG e. V., die in Potsdam, Campus Griebnitzsee, vom 28.-30. September 2017 stattfindet, verliehen.

\section{Bewerbung}

An die Bewerbung sind folgende Bedingungen geknüpft:

1. Die Bewerbung soll enthalten:

_Eine Zusammenstellung der wissenschaftlichen Arbeiten auf dem Gebiet der Adipositas, die Grundlage für die Preisverleihung sein sollen (ausgezeichnet werden heraus- ragende, publizierte, einschließlich zur Publikation eingereichte Forschungsarbeiten zu allen Aspekten der Adipositas),

- eine kurze Darstellung (= max. 1 Seite) der besonderen Bedeutung dieser Arbeiten für die AdipositasForschung,

_den Lebenslauf des Bewerbers,

_die Publikationsliste des Bewerbers,

-Sonderdrucke der drei wichtigsten Publikationen, sofern nicht in 1 . enthalten.

2. Das Alter der Bewerberin bzw. des Bewerbers muss unter 40 Jahren sein.

3. Die Bewerbungsfrist (Eingang) ist der 1. Juni 2017.

4. Die Bewerbungen sollen nur online an die Geschäftsstelle der DAG e. V., Frau Dr. Beatrix Feuerreiter, gesandt werden, E-Mail:

feuerreiter@adipositas-gesellschaft.de.

www.adipositas-gesellschaft.de 